\title{
A novel peptide motif binding to and blocking the intracellular activity of the human papillomavirus E6 oncoprotein
}

\author{
Susanne Dymalla • Martin Scheffner • Elvira Weber • \\ Peter Sehr • Claudia Lohrey • Felix Hoppe-Seyler • \\ Karin Hoppe-Seyler
}

\begin{abstract}
Specific types of human papillomaviruses (HPVs) cause cervical cancer. The viral E6 oncogene is a critical factor for maintaining the malignant phenotype of HPVpositive tumour cells. By yeast two-hybrid screening of a randomised peptide expression library, we isolated linear short peptides, which specifically bind to the HPV16 E6 oncoprotein. Sequence alignments and mutational analyses of the peptides identified a hitherto undiscovered E6-binding motif. Intracellular expression of a peptide containing the novel E6-binding motif resulted in inhibition of colony formation capacity, specifically of HPV16-positive cancer cells. A solubility-optimised variant of the peptide was created, which binds to HPV16 E6 with high affinity. Its intracellular expression efficiently induced apoptosis in HPV16-positive cancer cells. This was linked to restoration of intracellular p53 activities. Thus, this newly identified E6binding motif could form a novel basis for the development
\end{abstract}

S. Dymalla $\cdot$ C. Lohrey $\cdot$ F. Hoppe-Seyler $(\bowtie) \cdot$

K. Hoppe-Seyler $(\triangle)$

Molecular Therapy of Virus-Associated Cancers (F065),

German Cancer Research Center,

Im Neuenheimer Feld 242,

69120 Heidelberg, Germany

e-mail: hoppe-seyler@dkfz.de

e-mail: k.hoppe-seyler@dkfz.de

M. Scheffner $\cdot$ E. Weber

Department of Biology, University of Konstanz,

Konstanz, Germany

P. Sehr

Chemical Biology Core Facility, EMBL,

Heidelberg, Germany of rational strategies for the treatment of HPV16-positive preneoplastic and neoplastic lesions.

Keywords Human papillomavirus · Cervical cancer . Cancer therapy $\cdot$ Oncogenes

\section{Introduction}

Specific types of human papillomaviruses (HPVs) cause cervical cancer, the second most common cancer in females worldwide. In addition, oncogenic HPV types are associated with other cancers, e.g. in the anogenital region or oropharynx [1]. The most prevalent HPV type found in these cancers is HPV16, which, for example, accounts for $50-60 \%$ of all cervical carcinoma cases worldwide [2]. Recently developed prophylactic HPV vaccines hold great promise for a future prevention of HPV-associated cancers [3]. Yet, there are still many issues to be solved for a widespread application, which include high costs of the vaccine as well as problems related to medical infrastructure and health education [4]. The incidence of cervical cancer is estimated to increase by over $40 \%$ until the year 2020, mostly due to the population dynamics in the developing countries [5]. Thus, novel treatment strategies are urgently required.

The viral E6 oncogene is known to play a crucial role for the maintenance of the malignant phenotype of HPVpositive cancer cells [1]. Therefore, inhibition of E6 function could be ultimately developed into a rational anti-cancer strategy. Indeed, interference with E6 activity has been found to exert strong anti-oncogenic effects in HPV-positive cancer cells in vitro and in vivo, resulting either in apoptosis [6-10], growth arrest [11, 12] or both [13]. Since E6, as a non-cellular protein, is not expressed in 
undiseased cells, E6 inhibitors should principally allow a selective attack on HPV-positive cells. As proof of concept, previous studies have shown that specific targeting of E6 at the protein level by peptide aptamers or intracellular antibodies ("intrabodies") induced apoptosis in HPV-positive cancer cells, without affecting HPV-negative cells [6, 8, 10]. However, both peptide aptamers and intrabodies are relatively large and complex molecules, which, at current, restricts their further development into drugs [14, 15]. On the other hand, intracellular expression of peptides, which were rationally derived from the binding domains of natural E6 interaction partners, such as E6AP, failed to exert antioncogenic effects in HPV-positive cells [8].

Here, we screened a randomised expression library for small linear peptides targeting the HPV16 E6 oncoprotein. A hitherto unrecognised peptide-binding motif was identified, which specifically binds to the HPV16 E6 protein. Intracellular expression of peptides harboring the novel E6binding motif efficiently induced apoptosis, selectively in HPV16-positive cancer cells. On the basis of this bioactivity, this novel peptide motif should serve as a valuable new tool for the development of therapeutic strategies to specifically target HPV-positive preneoplastic and neoplastic lesions.

\section{Materials and methods}

Plasmids

Yeast expression vectors encoding full-length E6 proteins fused to the GAL4-DNA-binding domain were obtained by subcloning HPV16 E6, HPV18 E6, HPV6 E6 and HPV 11 E6 coding sequences into pPC97 [6]. For CheckMate ${ }^{\mathrm{TM}}$ (Promega, Mannheim, Germany) analyses, the complete HPV16 E6 coding sequence was fused to GAL4-BD in pBIND; peptide coding sequences were fused to VP16-AD in pACT. To monitor expression in mammalian cells, peptide sequences were fused to human recombinant green fluorescent protein (hrGFP; Stratagene, Heidelberg, Germany) in pCEP4 (Invitrogen, Karlsruhe, Germany). Mutant derivatives of pep11 were generated either by introducing double-stranded synthetic oligonucleotides of the desired sequence or by site directed mutagenesis. For ectopic expression in mammalian cells, HPV16 E6 was expressed from pcDNA3 (Invitrogen) and p53 from p53wt [16].

Cell lines and transfections

HPV16-positive SiHa, CaSki and HPV18-positive HeLa cervical carcinoma cells, H1299 lung cancer cells, U2OS osteosarcoma cells and MCF-7 breast cancer cells were maintained in Dulbecco's minimal essential medium
(pH 7.2), supplemented with $10 \%$ foetal calf serum (FCS). HPV16-positive MRI-H-186 cervical carcinoma cells [17] were grown in RPMI-1640 medium, supplemented with $10 \%$ FCS. Plasmids were transfected by calcium phosphate co-precipitation, as described [17], or with Fugene HD (Roche Diagnostics, Mannheim, Germany).

\section{Peptide screening}

Yeast strain KF1 (MATa trp1-901 leu2-3,112 his3-200 gal4A gal80A LYS2::GAL1-HIS3 GAL2-ADE2 met $2:$ : GAL7-lacZ SPAL10-URA3) contains three selectable nutritional marker genes under the control of GAL4-binding sites [6]. A peptide expression library was generated by fusing randomised 60mer oligonucleotides to GAL-AD in pADtrx [6], thereby replacing its trx insert. Oligonucleotides contained triplets of the sequence $\mathrm{NNK}$ (where $\mathrm{N}=\mathrm{G}$, $\mathrm{A}, \mathrm{T}$ or $\mathrm{C} ; \mathrm{K}=\mathrm{G}$ or $\mathrm{C}$ ), which encode for all 20 amino acids but result in only one stop codon. KF1 transformants expressing GAL4-BD-HPV16 E6 and randomised peptides were initially selected for activation of the intermediate stringent [6] marker GAL2-ADE. Subsequently, they were analysed by replica plating for activation of the GAL4dependent GAL1-HIS3, GAL2-ADE and SPO13-URA3 genes. Peptide expression vectors from clones, which activated at least two marker genes, were rescued, and activation of the selectable markers was verified by rescreening. E6APpep corresponds to amino acids 391-408 of E6AP [18] and served as a positive control for E6 binding.

Mammalian two-hybrid assays

The CheckMate ${ }^{\mathrm{TM}}$ system (Promega) was used to investigate the binding capacities of pep11 and pep11 mutants to HPV16 E6 in mammalian cells. Both pBIND and pACT fusion constructs were transfected into HeLa cells along with the GAL4-responsive luciferase reporter construct pG5luc and the internal standard pCMV-Gal [7]. Two days after transfection, cells were harvested. Luciferase activities were determined as duplicates in at least three independent experiments and normalised for pCMV-Gal activities, as described [19].

Protein purification, synthetic peptides

Recombinant GST-HPV16 E6 was expressed in Escherichia coli strain BL21 and purified as described [20]. Peptides were chemically synthesised with or without an N-terminal fluorescein label at the Peptide Synthesis Core Facility (German Cancer Research Center), using Fmoc chemistry. Crude peptides were purified by HPLC and checked by mass spectrometry. Peptides were dissolved in DMSO to obtain 
$10 \mathrm{mM}$ solutions, supplemented with equimolar DTT and stored at $-20^{\circ} \mathrm{C}$.

\section{Fluorescence polarisation}

For equilibrium binding studies, twofold serial dilutions of GST-HPV16 E6 were prepared in fluorescence polarisation (FP) buffer (PBS, 0.05\% Tween 20, 1 mM DTT) with varying glycerol contents to maintain a continuous glycerol concentration (14\% in the assay). Fluorescein-labelled peptides $(30 \mathrm{nM})$ were mixed with protein samples in black 384-well ProxiPlates (PerkinElmer, Boston, USA) to a final volume of $10 \mu \mathrm{l}$ and incubated for $90 \mathrm{~min}$ at room temperature. For competition studies, 1:3 serial dilutions of unlabelled peptides were co-incubated with $30 \mathrm{nM}$ of fluorescein-labelled peptides and $500 \mathrm{nM}$ of GST-HPV16 E6 in a final volume of $15 \mu \mathrm{l}$ for $90 \mathrm{~min}$. FP signals were measured in an Envision ${ }^{\mathrm{TM}}$ Multilabel Plate Reader (PerkinElmer). Background corrected signals were plotted against protein concentrations and fitted with a one-site saturation model (binding) or a non-linear regression model (competition) using SigmaPlot (Systat Software, San Jose, USA).

\section{Colony formation assays}

Cell lines transfected with hrGFP-peptide expression vectors were selected for the presence of pCEP4 by hygromycin B treatment. Colonies were fixed and stained with formaldehyde-crystal violet after 7-14 days of selection.

p53-Responsive reporter assays

hrGFP-peptide expression vectors were transfected into SiHa, MRI-H-186 or HeLa cells, together with the p53responsive reporter construct p53CON-pGUP.PA.8 [21] and pCMV-Gal as internal standard. Two days after transfection, cells were harvested and luciferase activities were determined as duplicates in at least three independent experiments, normalised for pCMV-Gal activities, as further described [19].

\section{Apoptosis assays}

Cells grown on coverslips were transfected with pCEP4hrGFP-peptide expression vectors and fixed with paraformaldehyde (4\% in PBS), 2 days after transfection. Terminal deoxynucleotidyltransferase-mediated UTP end labelling (TUNEL) analysis was performed, utilising the in situ cell death detection kit TMR red (Roche Diagnostics). DNA was stained with 4',6-diamidino-2-phenylindole (Roche Diagnostics). Apoptotic DNA strand breaks, peptide ex- pression and nuclei were visualised with a Vanox-T AH-2 epifluorescence microscope (Olympus, Center Valley, USA). Pictures were captured with an F-View camera (Soft Imaging System, Olympus) and analysed with analySIS ${ }^{\wedge} \mathrm{B}$ software (Soft Imaging System).

\section{Western blot analysis}

Two days after transfection, cellular protein was extracted in RIPA buffer (10 mM Tris- $\mathrm{HCl} \mathrm{pH} 7.5,150 \mathrm{mM} \mathrm{NaCl}$, $1 \mathrm{mM}$ EDTA, $1 \%$ NP40, $0.5 \%$ sodium deoxycholate, $0.1 \%$ SDS). Approximately $30 \mu \mathrm{g}$ of protein was separated by $12.5 \%$ SDS-PAGE, transferred to an Immobilon-P membrane (Millipore, Eschborn, Germany) and analysed by enhanced chemiluminescence (Amersham Biosciences, Freiburg, Germany). The following primary antibodies were used for protein detection: anti- $\beta$-galactosidase (Promega), anti- $\alpha$-tubulin CP06 (Calbiochem, Schwalbach, Germany), anti-hrGFP (kind gift of H. Zentgraf, Heidelberg), anti-p53 DO-1 (BD PharMingen, San Diego, USA), anti-p21 OP64 (Calbiochem) and anti-PUMA (Cell Signaling, Danvers, USA).

Ubiquitination assay

The HPV16 E6 protein was expressed as glutathione $S$-transferase fusion proteins in E. coli $\mathrm{DH} 5 \alpha$ as described [18]. The ubiquitin-activating enzyme E1, E6AP, and the ubiquitin-conjugating enzyme $\mathrm{UbcH} 5 \mathrm{~b}$ were expressed in the baculovirus system or in E. coli BL21 by using the pET expression system as described [22]. For in vitro ubiquitination of human Dlg [23], $1 \mu$ l of wheat germ extracttranslated ${ }^{35}$ S-labelled Dlg was incubated with $50 \mathrm{ng}$ E1, $50 \mathrm{ng} \mathrm{UbcH5}$, $50 \mathrm{ng}$ E6AP and $10 \mu \mathrm{g}$ ubiquitin (Sigma, Taufkirchen, Germany) in the absence or in the presence of bacterially expressed E6 (50 ng) and increasing amounts (micrograms) of the peptides indicated in 50- $\mu$ l volumes. In addition, reactions contained $25 \mathrm{mM}$ Tris- $\mathrm{HCl}(\mathrm{pH} 7.5)$, $60 \mathrm{mM} \mathrm{NaCl}, 3 \mathrm{mM}$ DTT, $2 \mathrm{mM}$ ATP and $4 \mathrm{mM} \mathrm{MgCl}_{2}$. After incubation at $30^{\circ} \mathrm{C}$ for $2 \mathrm{~h}$, total reaction mixtures were electrophoresed in $10 \%$ SDS-polyacrylamide gels and the ${ }^{35} \mathrm{~S}$-labelled substrates detected by fluorography using the LAS-3000 imaging system (Fujifilm, Düsseldorf, Germany). Quantitation of the intensity of the signals was performed with the Aida 4.08 Software package (Raytest, Straubenhardt, Germany).

\section{Results}

An expression library encoding randomised linear 20-mer peptides linked to the transcriptional activation domain of GAL4 was created (complexity approximately $8.3 \times 10^{6}$ ) 
and screened by yeast two-hybrid for molecules binding to the HPV16 E6 oncoprotein. Among $2.1 \times 10^{7}$ transformants initially screened for their ability to activate the $A D E 2$ marker, we isolated six different clones, which activated in replica analyses the HIS3 and ADE2 selection markers in yeast test strain KF1. In addition, three of them also stimulated the URA3 marker, which is activated in KF1 only by relatively strong protein/protein interactions [6].

Plasmids were isolated from the positive clones, and the DNA sequences encoding for the peptide inserts were determined (Fig. 1). Sequence comparison of the deduced peptide sequences showed that four of them (pep1, pep 5, pep10, pep22) exhibited homologies to the known consensus sequence for E6-binding peptides (Fig. 1), as previously defined from naturally occurring E6 interaction partners [24, 25]. Notably, however, three peptides contained a homologous novel motif, either alone (pep11) or in combination with the previously defined consensus motif (pep1, pep5). This new motif is characterised by the consensus sequence CXChXCh (Fig. 1) and clearly differs from the previously defined consensus sequence of E6-binding peptides (Fig. 1). In data bank searches, we did not find any obvious similarities of the new motif to sequences of naturally occurring E6-binding proteins or any human protein (not shown).

In order to assess the specificity of HPV16 E6/peptide interactions, binding analyses were performed in yeast and mammalian cells. None of the peptides was detectably bound to the structurally related E6 proteins of HPV6, HPV11 and HPV18 in yeast two-hybrid analyses (Table 1), with the exception of pep11, which very weakly activated the (most sensitive [6]) HIS3 marker in KF1 when co-

\begin{tabular}{lc} 
pep1 & KGSLNCSCLVCWLQMFLGEFGP \\
pep2 & GWECVLWSDFEWPCACWGLHGP \\
pep5 & PELLGCFCLCCWIECQIGSYGP \\
pep10 & SGGGDSFSWAMAFYFLDTLLGP \\
pep11 & EFGSGCSCIVCIGLI \\
pep22 & GMFTGESWVSSSLMVLDALLGP \\
\hline
\end{tabular}

$\begin{array}{lr}\text { novel motif } & \text { CXChXCh } \\ \text { E6AP motif } & \text { IbXhLsa }\end{array}$

Fig. 1 A novel consensus motif found in HPV16 E6-binding peptides. Sequences of the E6-binding peptides identified in this study are indicated. All randomised 20-mer peptides in the expression library contain (vector-derived) additional glycine and proline residues at the C-terminus, except for peptides prematurely terminated due to stop codons in their nucleotide sequence (e.g. the 15-mer pep11). Peptides are arranged to illustrate their homologies with the previously defined E6-binding consensus motif (indicated in blue), as deduced from natural E6 interaction partners (LbXhLsa; $X$ any amino acid, $h$ hydrophobic amino acid, $b$ amino acid that can form additional hydrogen bonds, $s$ small amino acid, $a$ acidic residue; modified from $[24,25])$. In addition, homologies of individual peptides to the newly identified ( $\mathrm{CXChXCh)} \mathrm{motiv} \mathrm{are} \mathrm{illustrated} \mathrm{(indicated} \mathrm{in} \mathrm{red)}$
Table 1 Binding specificity of HPV16 E6-binding peptides

\begin{tabular}{lllll}
\hline & $16 E 6$ & $18 \mathrm{E} 6$ & $6 \mathrm{E6}$ & $11 \mathrm{E6}$ \\
& HAU & HAU & HAU & HAU \\
\hline pep1 & +++ & --- & --- & --- \\
pep2 & +++ & --- & --- & --- \\
pep5 & +++ & --- & --- & --- \\
pep10 & ++- & --- & --- & --- \\
pep11 & ++- & $(+)--$ & --- & -- \\
pep22 & ++- & --- & --- & --- \\
E6APpep & ++- & ++- & --- & --- \\
\hline
\end{tabular}

Binding studies were performed in yeast strain KF1. $-/+$ indicates whether the marker genes GAL1-His3 (H), GAL2-ADE2 (A) or SPO13-URA3 (U) were activated

expressed with HPV18 E6. As expected, E6APpep, a control peptide corresponding to the binding domain of the natural E6-binding protein E6AP [18], interacted with both HPV16 and HPV18 E6 (Table 1).

In order to investigate whether the peptides can bind to HPV16 E6 in mammalian cells, the CheckMate ${ }^{\mathrm{TM}}$ system was employed. In this mammalian two-hybrid system, the interaction of two binding partners leads to the activation of a cotransfected luciferase reporter plasmid. As shown in Fig. 2, all peptides - with the exception of pep2-led to an activation of the reporter in HeLa cells when co-expressed with HPV16 E6. No interaction of pep11 with HPV18 E6 was detectable in mammalian cells (not shown). Taken together, these findings demonstrate that binding of the newly isolated peptides is highly specific for HPV16 E6 and-except for pep2 - is detectable in human cervical cancer cells.

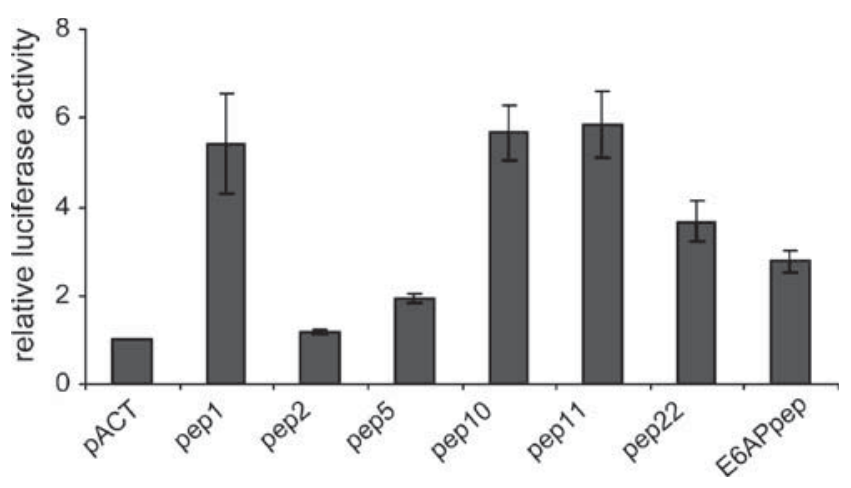

Fig. 2 Peptide binding to HPV16 E6 in mammalian cells. For CheckMate ${ }^{\mathrm{TM}}$ analyses, HeLa cells were co-transfected with vectors coding for HPV16 E6 linked to the GAL4-BD (pBIND) and individual peptides linked to the VP16-AD (pACT), together with a luciferase reporter under transcriptional control of GAL4-binding sites. Relative luciferase activities are indicated as fold activation above the values obtained for the co-expression of pBIND-16E6 and control vector pACT. E6APpep, positive control expressing the 18-mer E6-binding domain of E6AP, linked to VP16-AD. Standard deviations are indicated 
Next, we tested whether intracellular expression of the E6binding peptides affects the phenotype of HPV16-positive cancer cells. As shown in Fig. 3, pep11, which contains the newly defined E6-binding motif, strongly interfered with the colony formation capacity of HPV16-positive CaSki or SiHa cells. On the contrary, growth of control cells, such as HPV18-positive HeLa and HPV-negative MCF-7 (Fig. 3) or H1299 cells (not shown), was not affected by pep11. These findings show that the detrimental effect of pep11 expression on colony formation capacity is HPV16-specific and is not due to unspecific toxicity induced by pep 11 expression. In contrast to pep11, expression of E6APpep did not affect the growth of either HPV16- or HPV18-positive cancer cells, under the same experimental conditions.

In view of its pronounced bioactivity in HPV16-positive cancer cells, further work concentrated on pep11. In order to investigate whether pep11 can bind to HPV16 E6 in vitro, several attempts were made to chemically synthesise pep11. However, yields were very poor, and the peptide was hardly soluble in aqueous solution. Since pep11 is relatively hydrophobic, we decided to create a solubilityimproved pep11 variant, which retains binding to HPV16 E6. First, we defined the critical amino acids required for binding to HPV16 E6. We systematically replaced all amino acids within pep11 by alanine. Mammalian twohybrid analyses showed that mutation of pep11 amino acid residues $\mathrm{C} 6, \mathrm{C} 8, \mathrm{I} 9, \mathrm{C} 11$ and $\mathrm{I} 12$ within the newly defined E6-binding consensus sequence were strongly detrimental to its binding capacity to E6 (Fig. 4a). In addition, the hydrophobic C-terminus of pep11 contributed to binding, whereas mutations of the amino acids N-terminal of the consensus motif were generally well tolerated.

Next, we replaced several amino acids by more hydrophilic residues in order to improve solubility. Exchanged residues were selected for their similar side chain volumes, chemical similarity and maintenance of overall peptide charge. For example, cysteine was replaced by serine, which differs from cysteine only by a hydroxyl group instead of a thiol group, and isoleucine was replaced by glutamine, which both have similar side chain volumes. Figure $4 \mathrm{~b}$ summarises which amino acid replacements conserved E6 binding. Based on these data, all functional mutations were merged to yield a solubility-improved variant pep11* (Fig. 4c and d). In addition, we created a second pep11 variant, termed pep11**, by adding a four amino acid solubility enhancement tag [26] at the N-terminus of pep11*, KEKE, maintaining the overall peptide charge (Fig. 4d). Both solubility-improved pep11 variants, pep $11^{*}$ and pep $11^{* *}$, retained E6-binding activity (Fig. 4c), whereas the respective E6-binding-defective mutants pep $11 * \mathrm{~m}$ and pep $11^{* *} \mathrm{~m}$, containing three cysteine to serine conversions, served as negative controls for further experiments.

To address the question whether pep11** binds HPV16 E6 in vitro, the solubility-improved variant pep $11^{* *}$ was chemically synthesised and investigated for binding to purified HPV16 E6 by FP. FP-based equilibrium binding
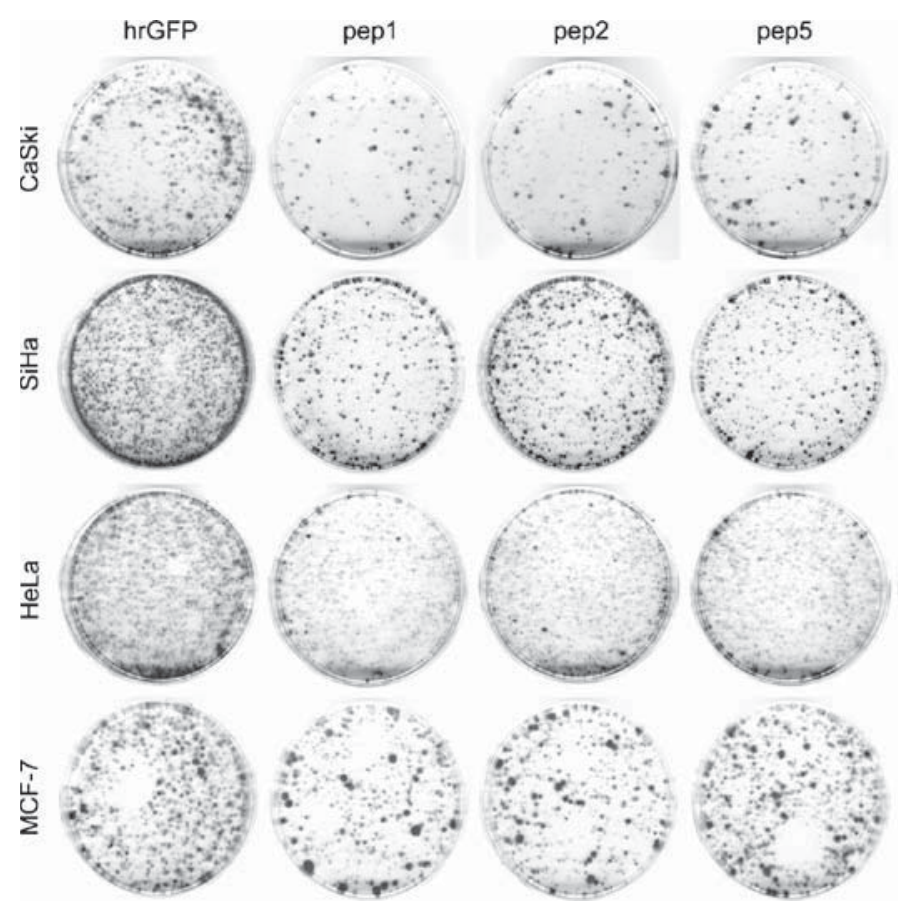

Fig. 3 pep11 specifically interferes with the colony formation capacity of HPV16-positive cells. Colony formation assays in HPV16-positive CaSki and SiHa as well as in HPV18-positive HeLa

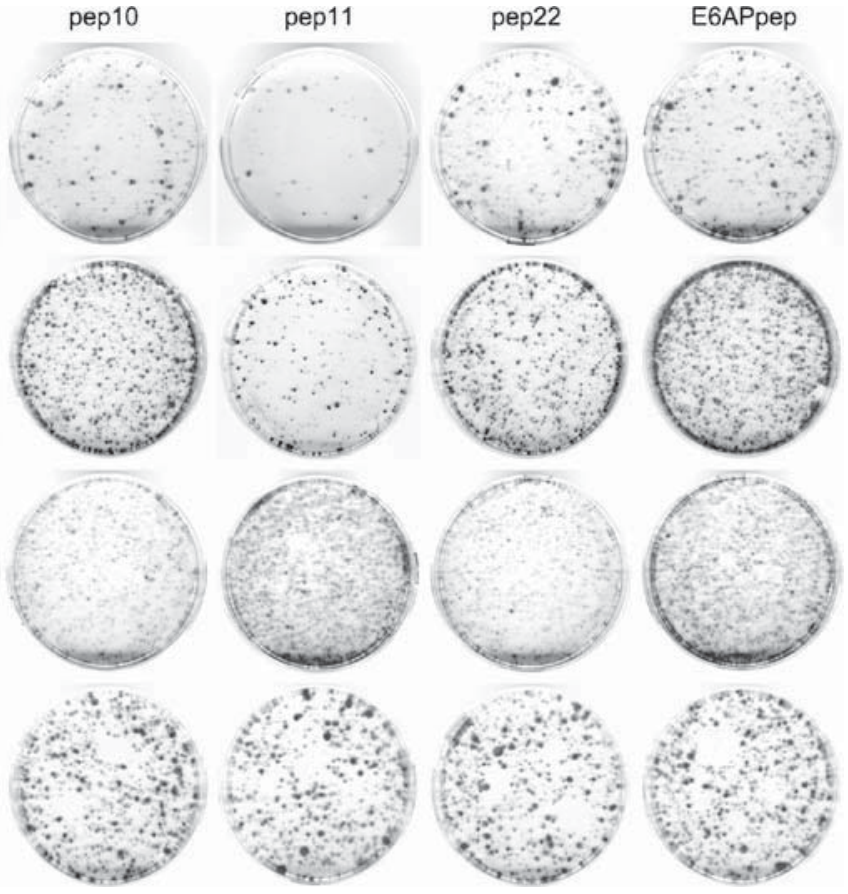

or HPV-negative MCF-7 cells, following transfection with hrGFPpeptide expression vectors. $h r G F P$ negative control 

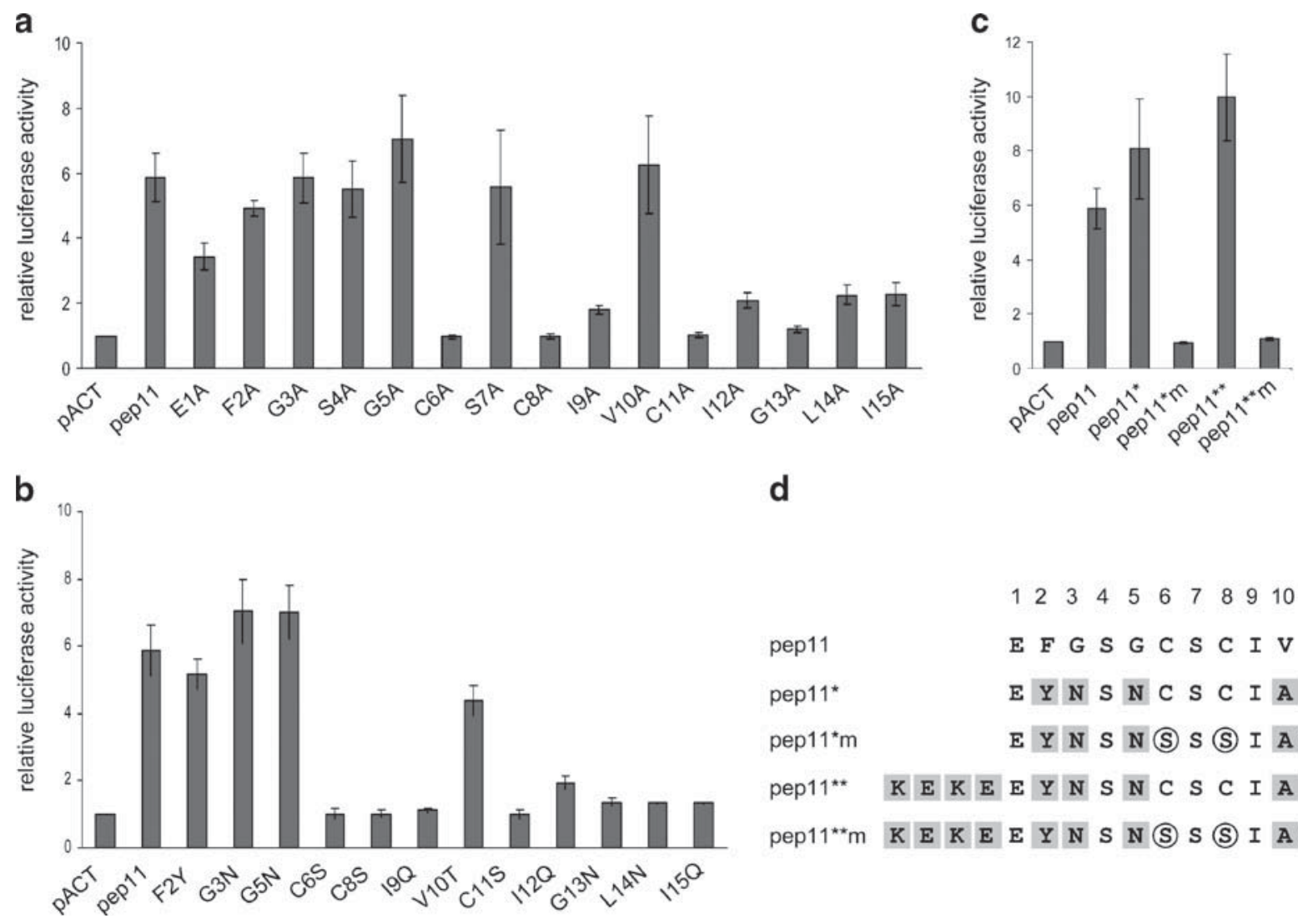

d

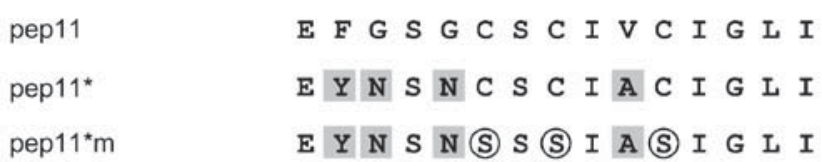

pep11** K E K E E Y N S N C S C I A C I G L I pep $11^{\star \star} \mathrm{m}$ K $\mathbf{E}$ K $\mathbf{E}$ E $\mathbf{Y}$ N S N (S) S (S) I A (S) I G I I

Fig. 4 Binding behaviour of pep11 variants to HPV16 E6. CheckMate $^{\mathrm{TM}}$ analyses in HeLa cells, co-transfected with expression vectors coding for GAL4-BD-HPV16 E6, VP16-AD-peptide variants and a GAL4-responsive luciferase reporter plasmid. Values represent fold luciferase activation above co-transfections with basic vector (pACT). $n=3 \pm$ SD. a Alanin scanning of pep11 residues critical for binding to HPV16 E6. b Solubility optimisation of pep11 by replacement of

individual amino acids by more hydrophilic residues. c Binding behaviour and $\mathbf{d}$ peptide sequences of solubility-optimised variants pep11* and pep11** and non-functional mutants pep11*m and pep11**m. Residues highlighted in grey indicate replacements in pep11 by more hydrophilic amino acids, which were not detrimental for binding. Circled residues indicate cysteine to serine conversions in the binding-defective mutants

studies (Fig. 5) revealed that pep11** associates with HPV16 E6 with high affinity $\left(K_{\mathrm{d}} 118 \mathrm{nM}\right)$. E6APpep bounds to E6 with an approximately five times lower affinity $(612 \mathrm{nM})$, comparable to the $K_{\mathrm{d}}$ value of $4 \mu \mathrm{M}$, determined previously by surface plasmon resonance [27].

As shown in Fig. 6a, alike pep11, the solubility-improved pep11** efficiently blocked colony growth of HPV16positive cancer cells (SiHa, MRI-H-186) but not of HPV16-negative control cells (HeLa, U2OS), showing that it is functional at the intracellular level. On the contrary, control peptide pep $11^{* *} \mathrm{~m}$ did not affect colony formation capacity of any of the tested cell lines. In order to assess whether pep $11^{* *}$-mediated inhibition of colony growth is linked to induction of apoptosis, TUNEL analyses were performed. As shown in Fig. 6b, expression of pep11**, but not of pep $11^{* *} \mathrm{~m}$, induced apoptosis. Again, this effect was specific for HPV16-positive cells in that HPV18-positive HeLa cells did not show signs of apoptosis upon intracellular pep $11^{* *}$ expression. Corresponding experiments analysing pep11* and pep11*m yielded identical results (not shown).

Since E6 induces the proteolytic degradation of p53 via formation of a trimeric complex with E6AP [18, 28], we investigated whether the pep11-induced phenotypic effects correlate with alterations of $\mathrm{p} 53$ protein levels. As shown in Fig. 7a, ectopic co-expression of p53 and HPV16 E6 in H1299 cells (in which the endogenous p53 gene is deleted) resulted in a strong reduction of $\mathrm{p} 53$ protein concentrations, as expected. The additional co-expression of pep11 or the solubility-optimised variants pep $11^{*}$ and pep $11^{* *}$ restored p53 protein levels, indicating interference with E6-mediated p53 degradation. In contrast, the E6-binding-defective mutant pep 1 *m did not replenish intracellular p53 levels. Corresponding results were obtained, analysing endogenous p53 levels in HPV16-positive MRI-H-186 cells. Again, p53 levels were increased by pep11 and even greater by the solubility-optimised pep $11^{*}$ and pep $11^{* *}$ variants, but not by pep $11^{*} \mathrm{~m}$ (Fig. 7b). Identical findings were obtained in HPV16-positive SiHa cells (not shown).

The increase in p53 upon expression of pep11 was linked to induction of known p53 target genes, such as p21 


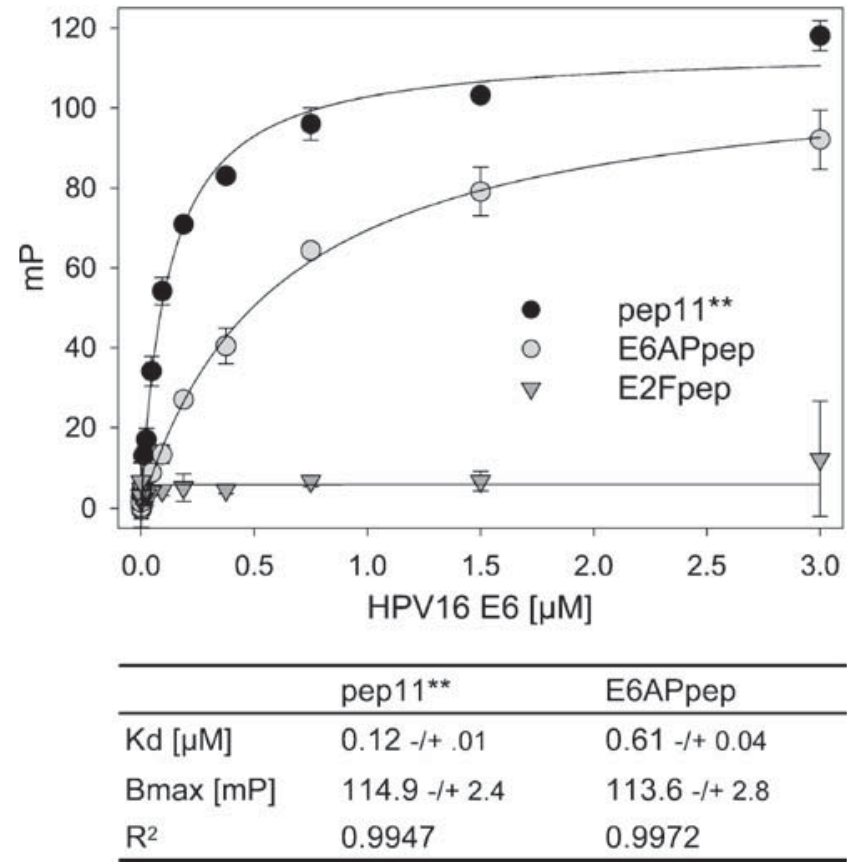

Fig. 5 FP equilibrium binding studies. Fluorescein-labelled peptides were incubated with increasing concentrations of purified GSTHPV16 E6. FP signals in $\mathrm{mP}$ units $(n=3)$ were plotted against protein concentrations (upper panel). $K_{\mathrm{d}}$ and Bmax values of E6/pep11** and E6/E6APpep interactions (lower panel). E2Fpep, negative control peptide, amino acids 409-426 (pRB-binding region) of E2F [37] or PUMA, indicating that the restored p53 protein is transcriptionally active (Fig. 7b). In line with this notion, pep11 and its solubility-improved variants, but not the respective E6-binding-defective mutants, induced a p53responsive promoter in transient transfection analyses in HPV16-positive SiHa, MRI-H-186 cells (Fig. 7c), or CaSki cells (not shown). On the contrary, all E6-binding competent pep11 variants did not influence p53 transcriptional activity in HPV18-positive HeLa (Fig. 7c) or HPV-negative U2OS (not shown) cells, further underlining their specific activity in HPV16-positive cells. These results indicated that the ability of different pep11 mutants to reactivate p53mediated transcription is closely linked to their ability to bind E6. This correlation was further substantiated by the analysis of a spectrum of additional pep11 mutants, showing a clear correlation between their capacity to bind E6 and the reconstitution of p53-mediated transcriptional activation, in HPV16-positive cancer cells (Table 2).

The reconstitution of intracellular p53 in HPV-positive cancer cells upon expression of pep11 raised the possibility that pep11 and its derivatives interfere with binding between E6 and E6AP. In an FP-based competition assay, we indeed observed that synthetic unlabelled pep $11^{* *}$ was capable of blocking the binding between HPV16 E6 and fluorescein-labelled E6APpep (Fig. 8a). In line with the differences in binding strength between E6/E6APpep and E6/pep11** (Fig. 5), pep11** was more efficient in competing for E6/E6APpep binding than an unlabelled a

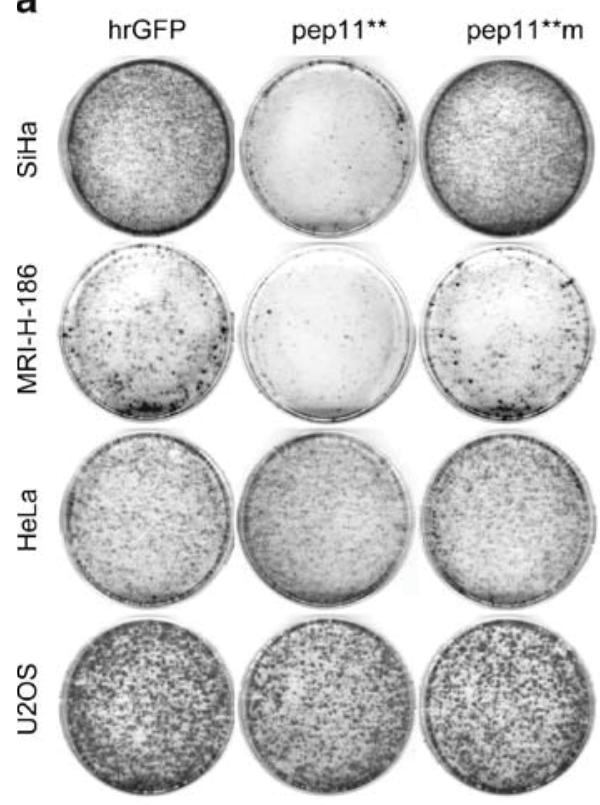

b

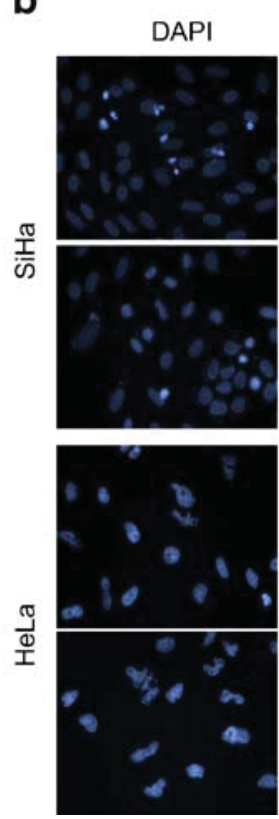

TUNEL
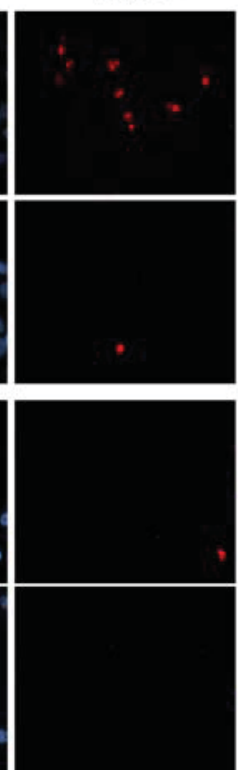

hrGFP
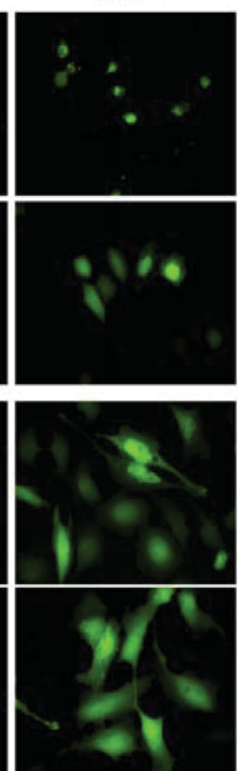

DAPI/hrGFP
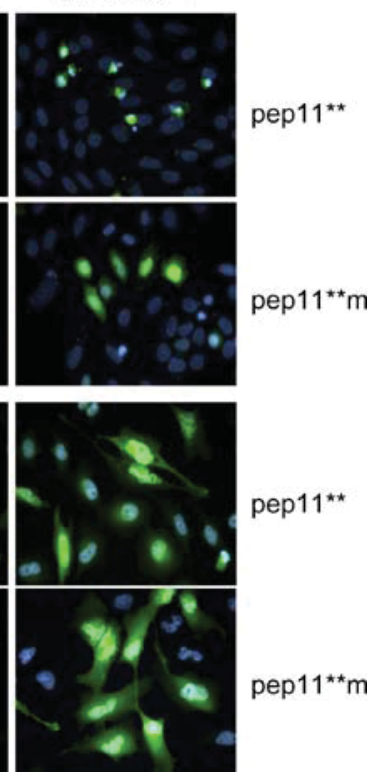

Fig. 6 pep11** blocks the growth of HPV16-positive cancer cells in clonogenic survival assays by inducing apoptosis. a Colony formation assays of HPV16-positive SiHa and MRI-H-186 cells, HPV18positive HeLa or HPV-negative U2OS cells, following transfection with hrGFP-pep11** and hrGFP-pep11**m expression vectors.
hrGFP negative control. b TUNEL assays in HPV16-positive SiHa and HPV18-positive HeLa cells upon transfection with the indicated hrGFP-peptide expression vectors. Note that pep $11^{* *}$ expressing $\mathrm{SiHa}$ cells, as visualised by GFP fluorescence, exhibit positive TUNEL staining 
a
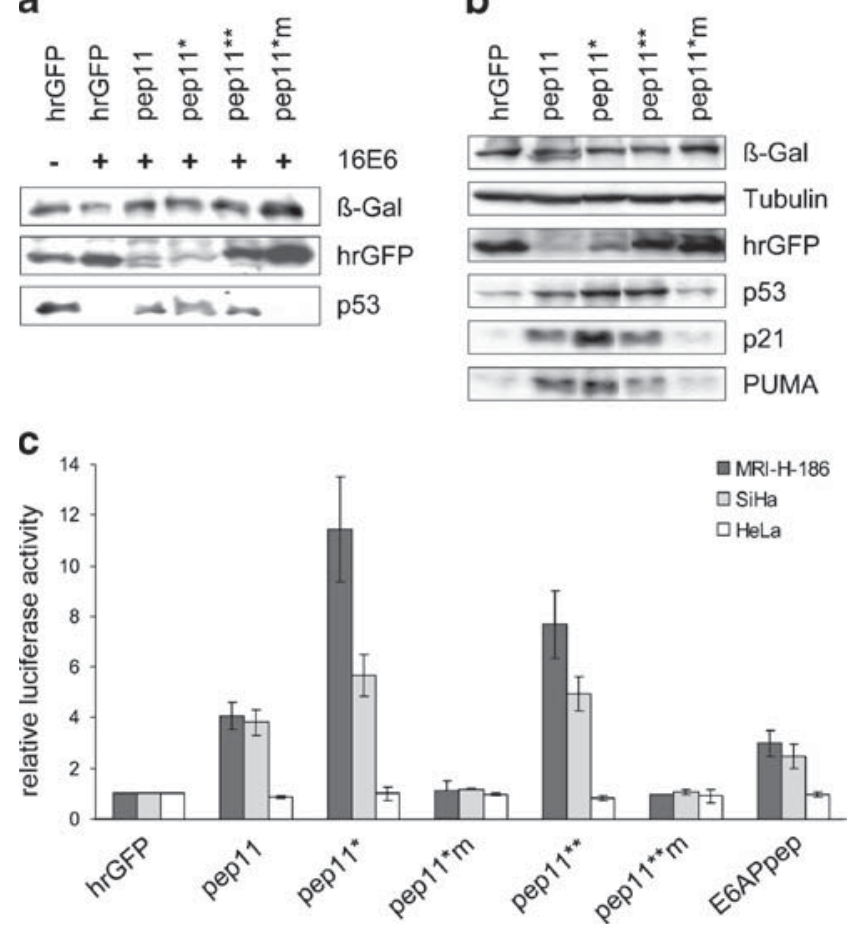

Fig. 7 Reconstitution of 553 by pep11 in HPV16 E6 expressing cells. a Western blot analysis upon co-expression of HPV16 E6 and p53 in H1299 cells - with an additional co-expression of hrGFP-pep11 variants, as indicated. $\beta$-Gal transfection control. b HPV16-positive MRI-H-186 cells expressing pep11 derivatives. Analysis of endogenous p53 levels as well as p21 and PUMA expression by Western blot. Tubulin loading control. c Luciferase reporter assays in HPV16positive MRI-H-186 and SiHa or in HPV18-positive HeLa control cells, upon co-transfection of a p53-responsive reporter plasmid together with expression vectors coding for different hrGFP-pep11 variants or hrGFP-E6APpep

E6APpep peptide (Fig. 8a). In order to investigate whether pep11 is also functional under in vitro conditions and may affect other E6 targets, we tested its effect on E6-induced ubiquitination of the human homologue of the Drosophila discs large $(\mathrm{hDlg})$ tumour suppressor protein, which is mediated by E6AP [23]. To this end, E6 was preincubated with increasing amounts of pep $11^{* *}$, and then, the mixture was added to a ubiquitination reaction containing E6AP and radiolabelled $\mathrm{hDlg}$. As shown in Fig. 8b, the presence of pep $11^{* *}$ efficiently interfered with E6-mediated ubiquitination of $\mathrm{hDlg}$. In contrast, the non-functional mutant pep11**m had no significant effect on $\mathrm{hDlg}$ ubiquitination, further corroborating the specificity of pep $11^{* *}$ activity.

\section{Discussion}

The present work identifies a novel peptide-binding motif, which specifically binds to the HPV16 E6 protein. Moreover, expression of this motif in the pep11 peptide
Table 2 Binding specificity and p53 reactivation pattern of pep 11 variants

\begin{tabular}{|c|c|c|}
\hline & HPV16 E6 binding & p53 reactivation \\
\hline pep11 wt & $5.87 \pm 0.76$ & $4.07 \pm 0.55$ \\
\hline E1A & $3.44 \pm 0.43$ & $3.70 \pm 0.53$ \\
\hline F2A & $4.92 \pm 0.25$ & $3.93 \pm 1.18$ \\
\hline G3A & $5.85 \pm 0.78$ & $4.15 \pm 1.55$ \\
\hline S4A & $5.51 \pm 0.86$ & $4.07 \pm 0.58$ \\
\hline G5A & $7.05 \pm 1.35$ & $3.80 \pm 0.51$ \\
\hline C6A & $0.97 \pm 0.06$ & $1.09 \pm 0.11$ \\
\hline S7A & $5.57 \pm 1.75$ & $4.94 \pm 0.96$ \\
\hline $\mathrm{C} 8 \mathrm{~A}$ & $0.99 \pm 0.08$ & $1.10 \pm 0.16$ \\
\hline I9A & $1.80 \pm 0.15$ & $3.15 \pm 0.44$ \\
\hline V10A & $6.25 \pm 1.49$ & $8.54 \pm 2.43$ \\
\hline C11A & $1.02 \pm 0.09$ & $0.94 \pm 0.19$ \\
\hline $\mathrm{I} 12 \mathrm{~A}$ & $2.07 \pm 0.24$ & $2.12 \pm 0.23$ \\
\hline G13A & $1.21 \pm 0.11$ & $1.16 \pm 0.16$ \\
\hline L14A & $2.26 \pm 0.29$ & $1.98 \pm 0.35$ \\
\hline $\mathrm{I} 15 \mathrm{~A}$ & $2.27 \pm 0.36$ & $1.95 \pm 0.25$ \\
\hline KEKE & $9.27 \pm 1.15$ & $5.06 \pm 0.92$ \\
\hline F2Y & $5.18 \pm 0.44$ & $5.17 \pm 0.32$ \\
\hline G3N & $7.04 \pm 0.96$ & $4.60 \pm 1.11$ \\
\hline G5N & $7.03 \pm 0.79$ & $4.07 \pm 0.90$ \\
\hline C6S & $1.03 \pm 0.15$ & $0.94 \pm 0.42$ \\
\hline $\mathrm{C} 8 \mathrm{~S}$ & $1.02 \pm 0.12$ & $1.05 \pm 0.57$ \\
\hline I9Q & $1.12 \pm 0.06$ & $1.43 \pm 0.10$ \\
\hline $\mathrm{V} 10 \mathrm{~T}$ & $4.39 \pm 0.45$ & $4.69 \pm 0.78$ \\
\hline C11S & $1.01 \pm 0.12$ & $1.11 \pm 0.54$ \\
\hline I12Q & $1.95 \pm 0.18$ & $2.86 \pm 0.88$ \\
\hline G13N & $1.36 \pm 0.11$ & $1.29 \pm 0.16$ \\
\hline $\mathrm{L} 14 \mathrm{~N}$ & $1.34 \pm 0.02$ & $1.23 \pm 0.14$ \\
\hline I15Q & $1.35 \pm 0.02$ & $1.19 \pm 0.17$ \\
\hline pep11* & $8.07 \pm 1.82$ & $11.44 \pm 2.09$ \\
\hline pep $11 * m$ & $0.94 \pm 0.03$ & $1.09 \pm 0.41$ \\
\hline pep $11^{* *}$ & $9.97 \pm 1.61$ & $7.68 \pm 1.31$ \\
\hline pep $11 * * m$ & $1.10 \pm 0.06$ & $0.98 \pm 0.01$ \\
\hline E6APрер & $2.77 \pm 0.23$ & $2.99 \pm 0.52$ \\
\hline
\end{tabular}

Binding of pep11 variants to HPV16 E6, as determined by CheckMate $^{\mathrm{TM}}$ analyses. Reactivation of endogenous $\mathrm{p} 53$ in HPV16-positive MRI-H-186 cells, as determined by p53-responsive luciferase assays upon expression of hrGFP-peptide fusions. Values indicate fold activation compared to co-transfecting the basic vectors (pACT for CheckMate $^{\mathrm{TM}}$ analyses, pCEP4-hrGFP for $\mathrm{p} 53$ reactivation assays). $n=3 \pm \mathrm{SD}$

restored functional p53 protein and specifically killed HPV16-positive cervical cancer cells by inducing apoptotic cell death. These findings support the idea that specific E6 inhibitors can efficiently induce cell death in HPV-positive cancer cells. Moreover, pep11 should provide a novel basis to generate pharmacologically useful molecules for the treatment of HPV-associated lesions.

The pronounced phenotypic effects observed in our study indicate that pep11 targets a surface domain on E6, which is critical for the anti-apoptotic activity of E6. In principle, this could be also expected for peptides 


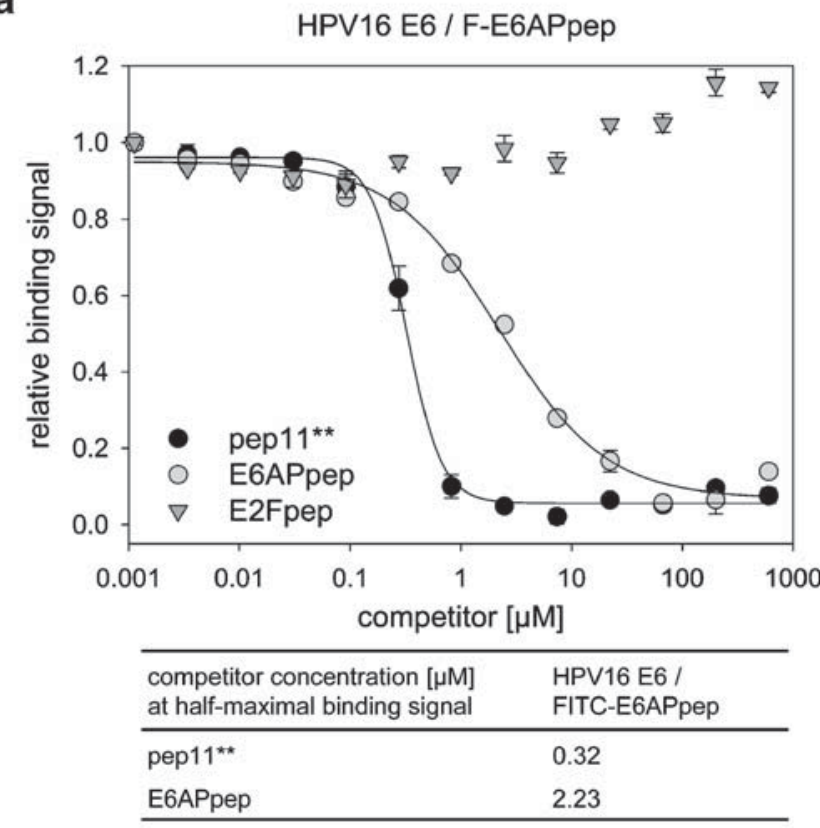

b
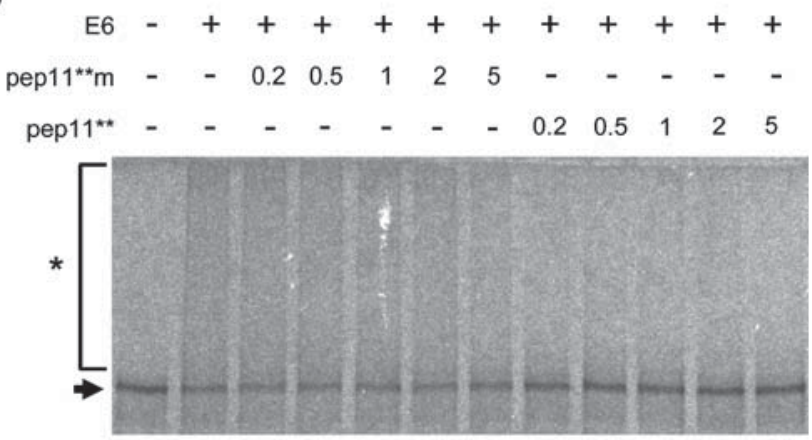

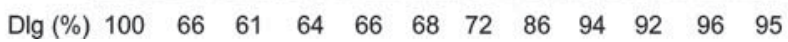

Fig. 8 pep11 competes for binding of E6APpep to E6 and interferes with the ubiquitination of hDlg. a Competition of HPV16E6-binding to fluorescein-labelled E6APpep with unlabelled competitor peptides pep11**, E6APpep or E2Fpep, analysed by FP. Relative FP signals are plotted against competitor concentrations. Concentrations of pep $11^{* *}$ and E6APpep, which yielded half-maximal binding signals are indicated. E2Fpep negative control. b E6-mediated ubiquitination of $\mathrm{hDlg}$ in vitro is inhibited by pep $11^{* *}$. In vitro wheat germ extracttranslated, radiolabelled $\mathrm{hDlg}$ was incubated in the absence or presence of HPV16 E6 and increasing amounts of the peptides pep $11^{* *}$ or control peptide pep $11^{* *} \mathrm{~m}$, as indicated, for $2 \mathrm{~h}$ under standard ubiquitination conditions. The reaction products were analysed by SDS-PAGE followed by fluorography and quantitation of the signal intensity of the band representing the non-modified form of hDlg. Running positions of the non-modified form (arrow) and the ubiquitinated forms (asterisk) of $\mathrm{hDlg}$ are indicated

corresponding to the E6AP-binding domain (E6APpep), which should have the potential to block E6-mediated degradation of the pro-apoptotic p53 protein. However, although E6APpep, or derivatives thereof, can interfere with p53 degradation in vitro [25, 29], they failed to exert detectable phenotypic effects upon intracellular expression in HPV-positive cancer cells [8]. In line and in stark contrast to pep11, we also did not observe a phenotypic effect exerted by E6APpep, when expressed under the same experimental conditions as pep11, in HPV-positive cancer cells.

The hydrophobic pep11 species isolated by yeast twohybrid screening was successfully rendered more hydrophilic by mutagenesis and by adding of a solubility enhancement tag. Whereas expression of pep11 was hardly detectable in mammalian cells, expression of both solubility-optimised versions, pep $11^{*}$ and pep $11^{* *}$, was easily demonstrated by Western blot and fluorescence-based analyses. In addition, solubility optimisation of pep 11 yielded a peptide variant applicable to chemical synthesis and suitable for subsequent biochemical studies.

Fluorescence polarisation analyses showed that the binding affinity of pep11** for E6 is considerably higher than that of E6APpep. In vitro competition assays further demonstrated that pep $11^{* *}$ efficiently blocked binding between HPV16 E6 and E6APpep. Vice versa, E6APpep competed with E6/pep11** binding, albeit with low efficiency (data not shown). This data could indicate that both peptides engage similar or at least partially overlapping binding sites on E6 with different binding affinities, which may provide an explanation for the increased bioactivity of pep11 in HPV16-positive cancer cells. However, since pep11 and E6AP exhibit different E6-binding motifs, it could alternatively be envisioned that pep11 targets a different binding domain on E6 than E6APpep and that pep11 may compete with E6AP for E6 binding by allosteric means or by structural disturbances of E6.

Mutational analyses indicated that three cysteine residues contained in pep11 were crucial for the binding to E6. In view of the fact that E6 itself also contains C-X-X-C motifs binding zinc [30], it could be considered that pep11 may bind to E6 via covalent linkage of cysteine residues or it may alter proper E6 folding by affecting zinc coordination of E6. Notably, several of previously isolated E6-binding peptide aptamers also contained C-X-X-C motifs [6]. Structural investigation of $\mathrm{E} 6$ has long been hindered by difficulties in purifying the protein in a stably folded form. Recently, advances in purifying the monomeric C-terminal part of HPV16 E6 have led to an NMR-based, three-dimensional model of E6 [31]. These data could be helpful to further explore the binding of pep11 to E6 and to define the critical amino acid residues and contact points. Work is in progress to study these questions in more detail. Notably, such structural data may also serve as a basis for in silico screening methods for small molecule pep11 mimics.

One important aspect for the bioactivity of pep 11 appears to be the reactivation of the p53 pathway, which is dormant in HPV-positive cancer cells due to the E6induced proteolytic degradation of p53 [28], but re- 
inducible upon repression of E6 expression [32, 33]. Indeed, apoptosis induction by pep11 was linked to a reconstitution of endogenous p53 protein levels in HPVpositive cancer cells, induction of p53 target genes and transcriptional stimulation of a p53-responsive reporter plasmid. Furthermore, analyses of a comprehensive series of pep11 mutants exhibited a clear correlation between the ability of individual peptides to bind HPV16 E6 and p53mediated transcriptional stimulation. The observation that pep11 interfered with E6-induced ubiquitination of $\mathrm{hDlg}$ indicates that pep11 also can affect additional tumour relevant E6 interactions, besides p53.

In view of its pronounced pro-apoptotic activity, it is conceivable that drugs acting similarly to pep 11 could be of therapeutic benefit for the treatment of HPV16-associated diseases. At least two avenues could be pursued in order to further develop the therapeutic potential of pep11 into an application perspective. Firstly, it could be envisioned to develop pep11 itself into a proteinaceous drug. Like protein therapy in general, this endeavour will require optimisation of both biopharmaceutic and pharmacokinetic parameters. For example, peptide stability could be increased by employing modified L-amino acids [34], stereochemical modifications of amino acids [35] or enhancement of peptide folds via intramolecular stabilisation [36].

Secondly, it will be interesting to explore whether it is possible to identify small, drug-like molecules from compound libraries, which can act as functional pep11 mimics. In this scenario, the pep11/E6 interaction should form a valuable basis for developing in vitro screening systems to identify compounds, which bind to the same, functionally critical E6 surface domain as targeted by pep11. The general feasibility of such "displacement screening" approaches has been demonstrated, for example, by identifying small molecules interfering with the interaction of peptide aptamers with their target proteins [15]. As for other in vitro analyses, such as NMR studies, the solubility-improved pep11 variant designed in the present study should also be useful for screening purposes. Conceivably, small molecules targeting the surface region defined by pep 11 could have the potential to also efficiently interfere with the anti-apoptotic activity of E6.

Acknowledgements This work was supported by the Deutsche Krebshilfe. We thank Angela Heilig for excellent technical assistance, Dr. H. Zentgraf for providing anti-hrGFP antibodies and Dr. Jennifer Reed for helpful discussions.

\section{References}

1. zur Hausen H (2002) Papillomaviruses and cancer: from basic studies to clinical application. Nat Rev Cancer 2:342-350
2. Schiffman M, Castle PE, Jeronimo J, Rodriguez AC, Wacholder S (2007) Human papillomavirus and cervical cancer. Lancet 370:890-907

3. Lowy DR, Schiller JT (2006) Prophylactic human papillomavirus vaccines. J Clin Invest 116:1167-1173

4. Agosti JM, Goldie SJ (2007) Introducing HPV vaccine in developing countries - key challenges and issues. New Engl J Med 356:1908-1910

5. Parkin DM, Bray F (2006) The burden of HPV-related cancers. Vaccine 24(Suppl. 3):S11-S25

6. Butz K, Denk C, Ullmann A, Scheffner M, Hoppe-Seyler F (2000) Induction of apoptosis in human papillomavirus-positive cancer cells by peptide aptamers targeting the viral E6 oncoprotein. Proc Natl Acad Sci U S A 97:6693-6697

7. Butz K, Ristriani T, Hengstermann A, Denk C, Scheffner M, Hoppe-Seyler F (2003) siRNA targeting of the viral E6 oncogene efficiently kills human papillomavirus-positive cancer cells. Oncogene 22:5938-5945

8. Griffin H, Elston R, Jackson D, Ansell K, Coleman M, Winter G, Doorbar J (2006) Inhibition of papillomavirus protein function in cervical cancer cells by intrabody targeting. J Mol Biol 355:360-378

9. Yamato K, Fen J, Kobuchi H, Nasu Y, Yamada T, Nishihara T, Ikeda Y, Kizaki M, Yoshinouchi M (2006) Induction of cell death in human papillomavirus 18-positive cervical cancer cells by E6 siRNA. Cancer Gene Ther 13:234-241

10. Lagrange M, Boulade-Ladame C, Mailly L, Weiss E, Orfanoudakis G, Deryckere F (2007) Intracellular scFvs against the viral E6 oncoprotein provoke apoptosis in human papillomavirus-positive cancer cells. Biochem Biophys Res Commun 361:487-492

11. Koivusalo R, Mialon A, Pitkänen H, Westermarck J, Hietanen S (2006) Activation of p53 in cervical cancer cells by human papillomavirus E6 RNA interference is transient, but can be sustained by inhibiting endogenous nuclear export-dependent $\mathrm{p} 53$ antagonists. Cancer Res 66:11817-11824

12. Courtete J, Sibler AP, Zeder-Lutz G, Dalkara D, Oulad-Abdelghani M, Zuber G, Weiss E (2007) Suppression of cervical carcinoma cell growth by intracytoplasmic codelivery of anti-oncoprotein E6 antibody and small interfering RNA. Mol Cancer Ther 6:1728-1735

13. DeFilippis RA, Goodwin EC, Wu L, DiMaio D (2003) Endogenous human papillomavirus E6 and E7 proteins differentially regulate proliferation, senescence, and apoptosis in HeLa cervical carcinoma cells. J Virol 77:1551-1563

14. Lobato MN, Rabbitts TH (2004) Intracellular antibodies as specific reagents for functional ablation: future therapeutic molecules. Curr Mol Med 4:519-528

15. Baines IC, Colas P (2006) Peptide aptamers as guides for smallmolecule drug discovery. Drug Discov Today 7-8:334-341

16. Hoppe-Seyler F, Butz K (1993) Repression of endogenous p53 transactivation function in HeLa cervical carcinoma cells by human papillomavirus type $16 \mathrm{E} 6$, human $\mathrm{mdm}-2$, and mutant p53. J Virol 67:3111-3117

17. Vogt M, Butz K, Dymalla S, Semzow J, Hoppe-Seyler F (2006) Inhibition of bax activity is crucial for the anti-apoptotic function of the human papillomavirus E6 oncoprotein. Oncogene 25:4009-4015

18. Huibregtse JM, Scheffner M, Howley PM (1993) Cloning and expression of the cDNA for E6-AP, a protein that mediates the interaction of the human papillomavirus E6 oncoprotein with p53. Mol Cell Biol 13:775-784

19. Butz K, Denk C, Fitscher B, Crnković-Mertens I, Ullmann A, Schröder CH, Hoppe-Seyler F (2001) Peptide aptamers targeting the hepatitis B virus core protein: a new generation of molecules with antiviral activity. Oncogene 20:6579-6586

20. Sehr P, Zumbach K, Pawlita M (2001) A generic capture ELISA for recombinant proteins fused to glutathione S-transferase: validation for HPV serology. J Immunol Methods 253:153-162 
21. Funk WD, Pak DT, Karas RH, Wright WE, Shay JW (1992) A transcriptionally active DNA-binding site for human p53 protein complexes. Mol Cell Biol 12:2866-2871

22. Nuber U, Schwarz S, Kaiser P, Schneider R, Scheffner M (1996) Cloning of human ubiquitin-conjugating enzymes UbcH6 and $\mathrm{UbcH} 7$ (E2-F1) and characterization of their interaction with E6AP and RSP5. J Biol Chem 271:2795-2800

23. Kuballa P, Matentzoglu K, Scheffner M (2007) The role of the ubiquitin ligase E6-AP in human papillomavirus E6-mediated degradation of PDZ domain-containing proteins. J Biol Chem 282:65-71

24. Chen JJ, Hong Y, Rustamzadeh E, Baleja JD, Androphy EJ (1998) Identification of an alpha helical motif sufficient for association with papillomavirus E6. J Biol Chem 273:13537-13544

25. Elston RC, Naphtine S, Doorbar J (1998) The identification of a conserved binding motif within human papillomavirus type $16 \mathrm{E} 6$ binding peptides, E6AP and E6BP. J Gen Virol 79:371-374

26. Kato A, Maki K, Ebina T, Kuwajima K, Soda K, Kuroda Y (2007) Mutational analysis of protein solubility enhancement using short peptide tags. Biopolymers 85:12-18

27. Zanier K, Charbonnier S, Baltzinger M, Nominé Y, Altschuh D, Travé G (2005) Kinetic analysis of the interactions of human papillomavirus E6 oncoproteins with the ubiquitin ligase E6AP using surface plasmon resonance. J Mol Biol 349(2):401-412

28. Scheffner M, Werness BA, Huibregtse JM, Levine AJ, Howley PM (1990) The E6 oncoprotein encoded by human papillomavirus types 16 and 18 promotes the degradation of p53. Cell 63:11291136

29. Sterlinko Grm H, Weber M, Elston R, McIntosh P, Griffin H, Banks L, Doorbar J (2004) Inhibition of E6-induced degradation of its cellular substrates by novel blocking peptides. J Mol Biol 335:971-985

30. Barbosa MS, Lowy DR, Schiller JT (1989) Papillomavirus polypeptides E6 and E7 are zinc-binding proteins. J Virol 63:1404-1407

31. Nominé Y, Masson M, Charbonnier S, Zanier K, Ristriani T, Deryckère F, Sibler AP, Desplancq D, Atkinson RA, Weiss E, Orfanoudakis G, Kieffer B, Travé G (2006) Structural and functional analysis of E6 oncoprotein: insights in the molecular pathways of human papillomavirus-mediated pathogenesis. Mol Cell 21:665-678

32. Butz K, Shahabeddin L, Geisen C, Spitkovsky D, Ullmann A, Hoppe-Seyler F (1995) Functional p53 protein in human papillomavirus-positive cancer cells. Oncogene 10:927-936

33. Goodwin EC, DiMaio D (2000) Repression of human papillomavirus oncogenes in HeLa cervical carcinoma cells causes the orderly reactivation of dormant tumor suppressor pathways. Proc Natl Acad Sci U S A 97:12513-12518

34. Fauchere JL, Thurieau C (1992) Evaluation of the stability of peptides and pseudopeptides as a tool in peptide drug design. Adv Drug Res 23:127-159

35. Fischer PM (2003) The design, synthesis and application of stereochemical and directional peptide isomers: a critical review. Curr Protein Pept Sci 4:339-356

36. Walensky LD, Kung AL, Escher I, Malia TJ, Barbuto S, Wright RD, Wagner G, Verdine GL, Korsmeyer SJ (2004) Activation of apoptosis in vivo by a hydrocarbon-stapled BH3 helix. Science 305:1466-1470

37. Helin K, Lees JA, Vidal M, Dyson N, Harlow E, Fattaey A (1992) A cDNA encoding a pRB-binding protein with properties of the transcription factor E2F. Cell 70:337-350 